\title{
Chromospheric impact of an exploding solar granule ${ }^{\star}$
}

\author{
C. E. Fischer ${ }^{1}$, N. Bello González ${ }^{1}$, and R. Rezaei ${ }^{2,3}$ \\ ${ }^{1}$ Kiepenheuer Institut für Sonnenphysik, Schöneckstrasse 6, 79104 Freiburg, Germany \\ e-mail: cfischer@kis. uni-freiburg.de \\ 2 Instituto de Astrofísica de Canarias, Avda Vía Láctea S/N, La Laguna 38200, Tenerife, Spain \\ 3 Departamento de Astrofísica, Universidad de La Laguna, 38205 La Laguna (Tenerife), Spain
}

Received 6 May 2017 / Accepted 24 May 2017

\begin{abstract}
Context. Observations of multi-wavelength and therefore height-dependent information following events throughout the solar atmosphere and unambiguously assigning a relation between these rapidly evolving layers are rare and difficult to obtain. Yet, they are crucial for our understanding of the physical processes that couple the different regimes in the solar atmosphere.

Aims. We characterize the exploding granule event with simultaneous observations of Hinode spectroplarimetric data in the solar photosphere and Hinode broadband Ca II H images combined with Interface Region Imaging Spectrograph (IRIS) slit spectra. We follow the evolution of an exploding granule and its connectivity throughout the atmosphere and analyze the dynamics of a magnetic element that has been affected by the abnormal granule.

Methods. In addition to magnetic flux maps we use a local correlation tracking method to infer the horizontal velocity flows in the photosphere and apply a wavelet analysis on several IRIS chromospheric emission features such as Mg II k2 $\mathrm{v}$ and Mg II k3 to detect oscillatory phenomena indicating wave propagation.

Results. During the vigorous expansion of the abnormal granule we detect radially outward horizontal flows, causing, together with the horizontal flows from the surrounding granules, the magnetic elements in the bordering intergranular lanes to be squeezed and elongated. In reaction to the squeezing, we detect a chromospheric intensity and velocity oscillation pulse which we identify as an upward traveling hot shock front propagating clearly through the IRIS spectral line diagnostics of Mg II h\&k.

Conclusions. Exploding granules can trigger upward-propagating shock fronts that dissipate in the chromosphere.
\end{abstract}

Key words. Sun: photosphere - Sun: chromosphere - Sun: oscillations

\section{Introduction}

Exploding granules are a readily seen event in the granulation pattern in the photosphere estimated to cover $2.5 \%$ of the solar surface at any given time (Namba 1986). They are bright granules that show a rapid expansion with expansion rates of 1.7 to $3.2 \mathrm{~km} \mathrm{~s}^{-1}$ and reach diameters as large as 5.5". Further, they show a dark core in the center, giving them the overall appearance of a bright ring before splitting into smaller granules (see e.g., Namba 1986; Rast 1995; Hirzberger et al. 1999). Their temporal evolution in intensity and velocity are well described in Roudier et al. (2001). More recently, Palacios et al. (2012) analyzed high-resolution spectropolarimetric IMaX/SUNRISE data and found that the evolution of several mesogranular-sized exploding granules is linked to magnetic flux emergence.

In the events described by Hirzberger et al. (1999) it becomes clear that the individual evolution of each exploding granule is shaped by the surrounding material and the conditions present when the granule expands. Often material is observed to be jammed at the borders of the exploding granule which cannot expand freely. With the high-resolution IRIS spectrograph recording the Mg II k\&h $2796 \AA$ spectral region, we are able to link the evolution of a magnetic element trapped by an exploding granule and the neighboring granules with the formation of a shock wave in the chromospheric layer.

\footnotetext{
* Movies associated to Figs. A.1 and A.2 are available in electronic form at http: //www . aanda. org
}

\section{Observations}

We analyzed data taken simultaneously with the Hinode (Tsuneta et al. 2008) and IRIS satellites (De Pontieu et al. 2014) on 28 April 2014 of a quiet Sun area at a heliocentric angle of $\mu=0.98$. The Hinode data consisted of binned Hinode Spectropolarimeter (SP) full Stokes $6302 \AA$ slit spectra (spatial sampling of $\left.\sim 0.32^{\prime \prime}\right)$ covering a region of about $9 \times 81 \operatorname{arcsec}^{2}$, spectral sampling of $21.5 \mathrm{~m} \AA$, and a cadence of $\sim 70 \mathrm{~s}$. The level 0 data were calibrated using the SolarSoft $s p \_p r e p . p r o$ routine (Lites \& Ichimoto 2013). The calibration routine also provides a file stksimg.sav with maps for the apparent magnetic flux densities ( $B_{\text {long }}^{\text {app }}$ and $\left.B_{\text {trans }}^{\text {app }}\right)$, as well as continuum maps constructed from the continuum level of the Stokes $I$ spectra (Lites et al. 2008). Ca II H images taken with the Hinode Broadband Filter Imager (BFI) at a cadence of $31 \mathrm{~s}$, exposure time of $0.3 \mathrm{~s}$, a field-of-view (FOV) of $38 \times 89 \mathrm{arcsec}^{2}$, and a spatial sampling $\sim 0.109^{\prime \prime}$ were processed using the $f g \_$prep.pro routine.

The IRIS satellite provided a slitjaw image time series in the $2796 \AA$ a band at a spatial sampling of $0.166^{\prime \prime}$, a FOV of $62 \times 66 \operatorname{arcsec}^{2}$ and a cadence of $19 \mathrm{~s}$. Slit spectra were obtained in a two-step raster (IRIS-program: medium sparse 2-step raster) with a cadence of $19 \mathrm{~s}$ for each of the two slit positions recording FUV and NUV spectra in several wavelengths of which we show results in the region of $\mathrm{Mg}$ II h\&k at $2796 \AA$. The spatial sampling along the slit was $0.166^{\prime \prime}$ and the step size of the $0.33^{\prime \prime}$ wide slit was $1^{\prime \prime}$. We obtain level 2 data by running the iris_make_lev2.pro code. The code performs dark and flat 

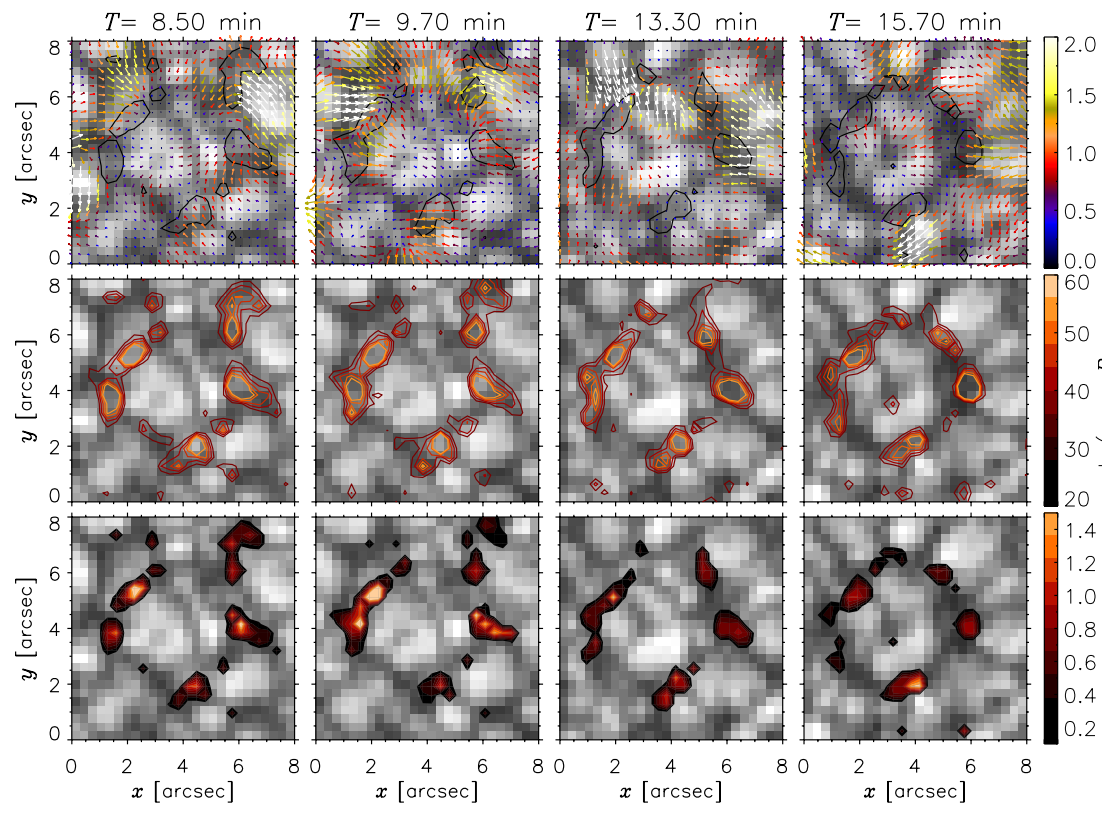

Fig. 1. Rows, top to bottom: first row: Hinode SP continuum images with the 2D field of the apparent horizontal velocities calculated by the local correlation tracking (LCT) method shown with colored arrows. Black contours denote $B_{\text {long }}^{\text {app }}$ at $\pm 30 \mathrm{Mx} \mathrm{cm}^{-2}$ and the times above the panels refer to the IRIS time line. In the second row the continuum images are now overplotted by contours of the absolute of $B_{\text {long }}^{\text {app }}$ magnetic flux density from absolute $20 \mathrm{Mx} \mathrm{cm}^{-2}$ to $60 \mathrm{Mx} \mathrm{cm}^{-2}$ in steps of $10 \mathrm{Mx} \mathrm{cm}^{-2}$. The last row shows the same continuum images now with filled contours of the magnetic field strength obtained from MERLIN inversions obtained with the default inversion parameters from http: //www2 . hao. ucar . edu/csac. calibration as well as an initial alignment between the slitjaw images and the spectra.

The data from both instruments were aligned using strong network magnetic elements appearing as brightenings in the Hinode $\mathrm{Ca}$ II $\mathrm{H}$ images and IRIS slitjaw images and rebinned to the IRIS slitjaw pixel size of $0.166^{\prime \prime}$. We created data cubes at the cadence of the IRIS slitjaw images by selecting for all instruments for each IRIS slitjaw timestamp the images closest to this timestamp. We did not perform an interpolation in time, which leads to repetition in the images for the cubes with a lower cadence (as seen in the online movies). The maximum error $\delta T$ in assigning the frames in reference to the IRIS slitjaw images (fastest cadence) can arise for the Hinode SP and is of the order of one minute.

\section{Results and discussion}

In the first panel of the movie attached to Fig. A.1 one can follow the temporal evolution of the exploding granule as seen in the constructed Hinode SP continuum maps. The time $T=$ 0 min refers to the beginning of the IRIS sequence starting at 11:39:36 UT. A small bright granule (area of $1.35 \operatorname{arcsec}^{2}$ ) is seen in the Hinode SP continuum map cutout located at $x=5^{\prime \prime}$ and $y=4.5^{\prime \prime}$ just two and a half minutes into the co-observing sequence. The granule explodes by expanding with a rate of about $\sim 0.77 \operatorname{arcsec}^{2}$ per minute, and then develops a dark core before splitting into several fragments, finally reaching a ring shaped form with an overall size of about $14 \operatorname{arcsec}^{2}$ at $T=$ 19 min. The morphological evolution of our exploding granule in the photosphere is consistent with Hirzberger et al. (1999).

There is a group of mixed polarity strong magnetic elements $(>500 \mathrm{G})$ that occupies the intergranular lanes in the vicinity of the exploding granule. After the exploding phase they are arranged in a circle around the granule, outlining its border and tracing the same ring-like pattern as seen in the Hinode SP continuum maps. The magnetic elements appear as bright points in the $\mathrm{Ca}$ II $\mathrm{H}$ images (third panel in the movie) which again line up as a chain encompassing the exploded granule at the end of the sequence. The bright points are also visible in the IRIS $2796 \AA$ band slitjaw images (last panel), as is the location of the slit at the edge of the exploding granule. By this point the interior features several dark lanes and after the maximum size is reached (at about 19-20 min) the shape looses coherency and, after a few minutes of granular evolution, cannot be traced anymore.

We use the Fourier local-correlation-tracking (FLCT) code (Fisher \& Welsch 2008) to obtain two-dimensional (2D) velocity fields inferred from the Hinode SOT SP continuum images. These continuum images were constructed using the continuum intensity in Stokes $I$ for each slit position and building up the map with a cadence for the entire FOV of about $70 \mathrm{~s}$. We set the full width at half maximum (FWHM) of the Gaussian used to weigh the pixels for the cross-correlation to four pixels $\left(1.2^{\prime \prime}\right)$. Running the code on pairs of two subsequent images of the time series, we obtain the apparent horizontal velocities for structures on a granular scale. The obtained results displayed in Fig. 1 show velocities greater than $1.5 \mathrm{~km} \mathrm{~s}^{-1}$ from the outer granules impinging on the exploding granule. We note that these velocities are only apparent horizontal velocities and are highly dependent on the structure scale of the image and the choice of FWHM of the employed Gaussian weighing function. Especially in a bright expanding larger region, the velocities might be underestimated in the interior due to no apparent structure changes. At $T=9.7 \mathrm{~min}$, the magnetic elements at the edge of the exploding granule at around $x=2^{\prime \prime}$ and $y=5^{\prime \prime}$ experience strong horizontal velocities from both sides; the interior of the exploding granule and the surrounding granules. This gives the impression of the magnetic elements being squeezed by the opposing flows. This is confirmed by the magnetic flux density evolution. The contours of the absolute $B_{\text {long }}^{\text {app }}$ maps show a stretching out of the magnetic elements, leading to a more elongated shape (compare, for example, panel one at $T=8.5 \mathrm{~min}$ and panel three at $T=13.3 \mathrm{~min}$ in Fig. 1) and separation of the magnetic flux elements. This is accompanied by a decrease in the total magnetic field strength as seen in the last row of Fig. 1 and is therefore not due to a change of the inclination angle tilting the magnetic field.

Figure 2 shows the time development of the (high) photospheric line-of-sight velocity from fits to the Fe I $2799.9 \AA$ line and the IRIS Mg II k2v peak intensity (a good indicator of the chromospheric temperature according to Pereira et al. 2013) in a spatial section along the slit where blacked-out pixels indicate locations where the feature finding failed. In both diagnostics, 


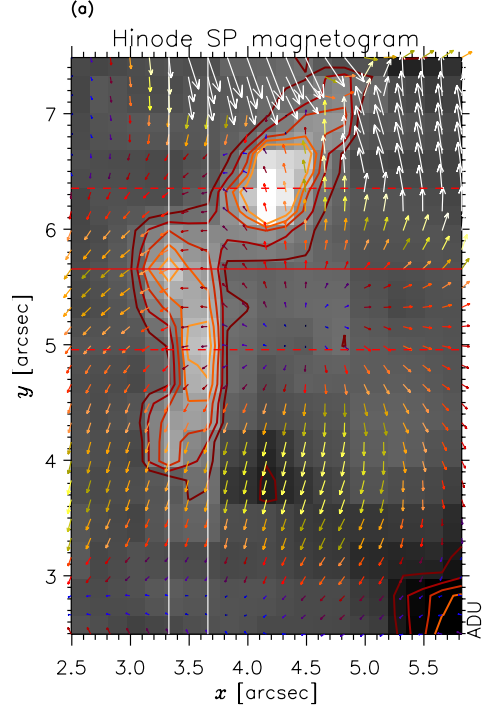

(b)

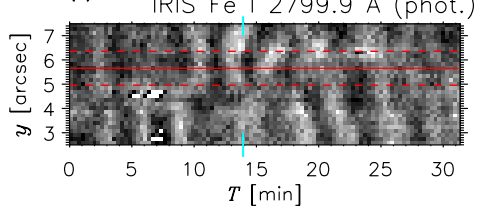

(c)
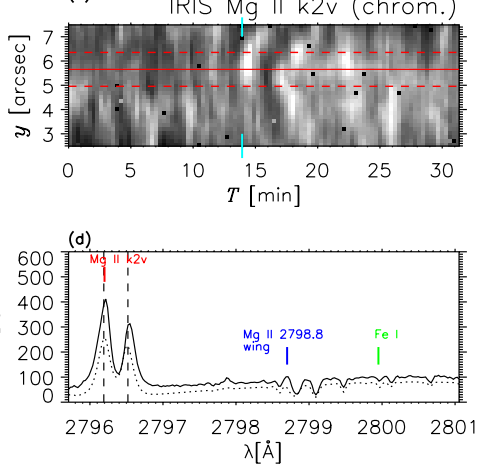

SLIT 2 Mg II k spectrum

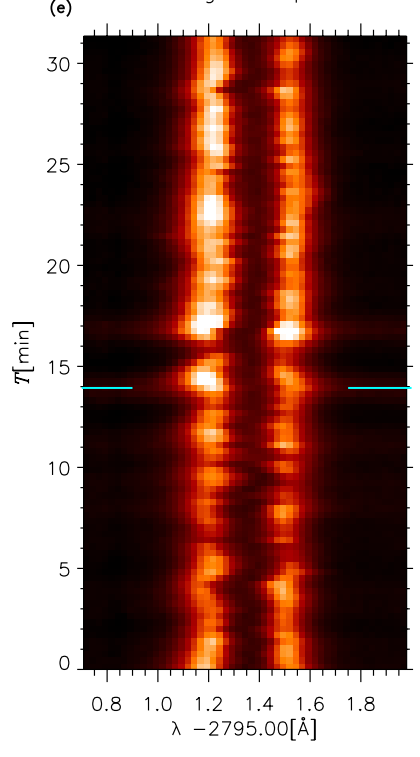

Fig. 2. Left to right: panel a): Hinode SP $B_{\text {long }}^{\text {app }}$ map now rebinned to the IRIS slitjaw scale with the same contours as in the second row of Fig. 1 and the horizontal flows indicated with colored arrows as in Fig. 1. The two vertical white lines delimit the position of the IRIS slit in use. Two dashed red horizontal lines and a solid red line mark a region of interest. Panels b) and c) are time-space images derived from spectra recorded by the second IRIS slit. Panel b): line-core velocity of the Fe I $2799.9 \AA$ line $\left( \pm 1.9 \mathrm{~km} \mathrm{~s}^{-1}\right.$, where down flows are positive $)$ retrieved with the MOSiC

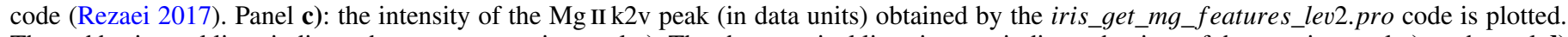
The red horizontal lines indicate the same area as in panel a). The short vertical lines in cyan indicate the time of the map in panel a), and panel d) shows a spectrum at that time (solid) and average quiet Sun spectrum (dotted). The dashed vertical lines denote the average position of the Mg II $\mathrm{k} 2$ peaks. The right panel demonstrates the temporal evolution of the IRIS slit spectra at the slit location marked by the solid red horizontal line in panels a) to c) with the time of panel a) indicated again with lines in cyan.

a similar oscillatory pattern is seen, although out of phase, and starting at around $T=10 \mathrm{~min}$ for the photosphere and around 12 min for the chromosphere lasting for a few minutes and located at the area $\pm 0.7^{\prime \prime}$ of the magnetic element location observed in the Hinode SP magnetic flux density maps. The oscillatory pattern appears one to two minutes after the previously described squeezing of the magnetic elements. As seen in the sample spectra in panel (d) we observe the wing of the Mg II 2798.8 A subordinate line going into emission. According to Pereira et al. (2015) this is a sign of heated material in the low chromosphere with cooler material above; a rare quiet Sun event (1\% of pixels) also observed in simulations (see also Carlsson et al. 2015). Schmidt \& Fisher (2013) have observed similarly rare emission in the Fe I $3969 \AA$ line out of phase with the simultaneously observed $\mathrm{Ca}$ II $\mathrm{H} 2 \mathrm{v}$ intensity as a marker of chromospheric dynamics.

Panel (e) in Fig. 2 shows the Mg II k spectra in time for the slit position marked with a red solid line in the previous panels. After an initial amplitude increase of the $\mathrm{Mg}$ II $\mathrm{k} 2 \mathrm{v}$ peak at about $11.5 \mathrm{~min}$, a dramatic sudden amplitude increase occurs at around $14 \mathrm{~min}$. At $T=17 \mathrm{~min}$ the red emission peak $\mathrm{Mg}$ II k2r brightens followed again by an increase of the $\mathrm{Mg}$ II k2 $\mathrm{v}$ peak. We find a switching of the amplitude increases in the two $\mathrm{k} 2$ peaks (see spectrum between $T=14.52 \mathrm{~min}$ and $T=16.78 \mathrm{~min}$ ). The ratio of the $\mathrm{Mg}$ II k2 $\mathrm{v}$ to $\mathrm{Mg}$ II $\mathrm{k} 2 \mathrm{r}$ peaks is a good indicator of the chromospheric velocity above the peak formation height (Pereira et al. 2013) with a higher peak in the blue emission peak corresponding to down flows. The movie attached to Fig. A.2 shows the detailed temporal evolution of the spectra with an increase of the near continuum compared to the average profile clearly seen, for example, at $T=16.78 \mathrm{~min}$ with the entire spectrum being shifted to higher intensity. These findings indicate a hot temperature front traveling through the high photosphere and throughout the low to mid chromosphere. This is compatible with a shock wave resulting from an oscillation induced at the location of the squeezed magnetic element.

We apply a wavelet analysis on various parameters derived from the IRIS slit spectra using the code provided by $\mathrm{C}$. Torrence and G. Compo ${ }^{1}$ using the default Morlet wavelet. In Fig. 3 we show the results for the velocity and intensity fluctuations in 1) the photospheric Fe I $2799.9 \AA$ line, 2) the MgIIh1 minimum, 3) the Mg II k2v peak sampling the low to mid chromosphere (Pereira et al. 2013), and 4) the Mg II k3 sampling the high chromosphere (Leenaarts et al. 2013). The wavelet analysis shows a power peak in both the velocity and intensity fluctuations of the photosphere to mid-chromosphere diagnostics, with a delay increasing with height between the maximum power. The maximum power is located at a period of between 2 and $3 \mathrm{~min}$ including also larger periods in the mid chromosphere of up to 5 to $6 \mathrm{~min}$. The high chromospheric signatures, as seen with the Mg II k3 amplitude (rightmost panel), show no power and only a weak response in the velocity power. As seen in the left panel mapping the amplitude in intensity, there is an increase visible in the amplitude which implies a change of the transition region height (Pereira et al. 2013). This could signify dissipation of the shock wave energy and local heating of the high chromosphere. Upward-propagating shock fronts are common in quiet Sun chromosphere, both in observations (Beck et al. 2008) and numerical simulations (Carlsson \& Stein 1997; Wedemeyer et al. 2004). We do not find a significant excess emission co-spatial with the chromospheric emission in the C II $1335 \AA$ line of IRIS, which is consistent with our finding that dissipation happens at the middle chromosphere.

1 http://atoc.colorado.edu/research/wavelets/ 

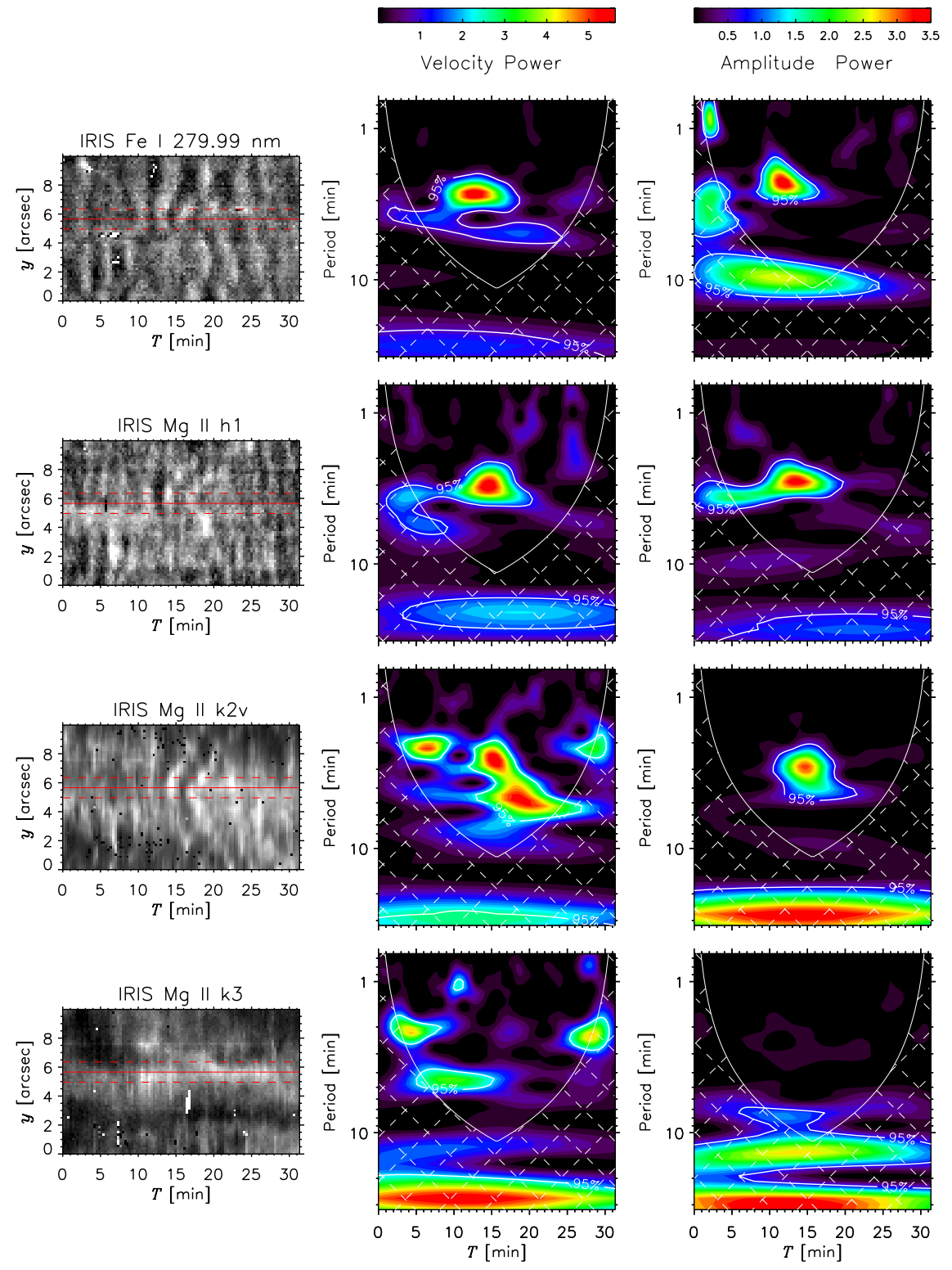

Fig. 3. Left to right: upper row: a time-space image retrieved from the velocity parameter of the Fe I $2799.9 \AA$ line $\left( \pm 1.9 \mathrm{~km} \mathrm{~s}^{-1}\right)$ obtained from the IRIS slit spectra using the MOSiC code. The dashed and solid horizontal red lines mark again the same region as in Fig. 2. The next two panels show the power for the velocity and the intensity fluctuations at periods ranging from $0.6 \mathrm{~min}$ to $21 \mathrm{~min}$ at the slit location marked with the red solid horizontal line in the first panel. The power is shown in a logarithmic scale with the hashed area marking periods in which the wavelet analysis yields untrustworthy results. The results obtained with a confidence level of $95 \%$ are contoured with a white solid line (not all are labeled). The following rows are in the same format as the first row, now showing first maps of the $\mathrm{Mg}$ II h1 minimum obtained through the MOSiC code and the $\mathrm{Mg}$ II k2v peak and the Mg II k3 core obtained with the iris_get_mg_features_lev2.pro code, where blacked out or white pixels signify a failed feature finding. The power for the velocity and the intensity fluctuations for these parameters are shown in the second and third panels in each row.

\section{Summary and conclusion}

A magnetic element at the edge of an exploding granule is squeezed by opposing horizontal flows, resulting in an elongation of the isocontours in magnetic flux as seen with the Hinode $\mathrm{SP}$, with a chromospheric response consequently being triggered and observed in the IRIS spectra. Signatures of an energy deposit in the middle chromosphere are seen through a wavelet analysis of different spectral features in the $\mathrm{Mg}$ II h \& k spectra. Our finding is consistent with an upward-propagating shock front triggered by the exploding granule.

Acknowledgements. C.E.F. has been funded by the DFG project Fi- 2059/1-1. N.B.G. acknowledges financial support by the Senatsausschuss of the LeibnizGemeinschaft, Ref.-No. SAW-2012-KIS-5 within the CASSDA project. R.R. acknowledges financial support by the Spanish Ministry of Economy and Competitiveness through project AYA2014-60476-P. Wolfgang Schmidt has provided important and useful comments on the manuscript. Hinode is a Japanese mission developed and launched by ISAS/JAXA, collaborating with NAOJ as a domestic partner, NASA and STFC (UK) as international partners. IRIS is a NASA small explorer mission developed and operated by LMSAL with mission operations executed at NASA Ames Research center and major contributions to downlink communications funded by ESA and the Norwegian Space Centre.

\section{References}

Beck, C., Schmidt, W., Rezaei, R., \& Rammacher, W. 2008, A\&A, 479, 213 Carlsson, M., \& Stein, R. F. 1997, ApJ, 481, 500

Carlsson, M., Leenaarts, J., \& De Pontieu, B. 2015, ApJ, 809, L30

De Pontieu, B., Title, A. M., Lemen, J. R., et al. 2014, Sol. Phys., 289, 2733

Fisher, G. H., \& Welsch, B. T. 2008, ASP Conf. Ser., 383, 373

Hirzberger, J., Bonet, J. A., Vázquez, M., \& Hanslmeier, A. 1999, ApJ, 527, 405

Leenaarts, J., Pereira, T. M. D., Carlsson, M., Uitenbroek, H., \& De Pontieu, B. 2013, ApJ, 772, 90

Lites, B. W., \& Ichimoto, K. 2013, Sol. Phys., 283, 601

Lites, B. W., Kubo, M., Socas-Navarro, H., et al. 2008, ApJ, 672, 1237

Namba, O. 1986, A\&A, 161, 31

Palacios, J., Blanco Rodríguez, J., Vargas Domínguez, S., et al. 2012, A\&A, 537, A21

Pereira, T. M. D., Leenaarts, J., De Pontieu, B., Carlsson, M., \& Uitenbroek, H. 2013, ApJ, 778, 143

Pereira, T. M. D., Carlsson, M., De Pontieu, B., \& Hansteen, V. 2015, ApJ, 806, 14

Rast, M. P. 1995, ApJ, 443, 863

Rezaei, R. 2017, ArXiv e-prints [arXiv: 1701.04421]

Roudier, T., Eibe, M. T., Malherbe, J. M., et al. 2001, A\&A, 368, 652

Schmidt, W., \& Fisher, J. 2013, A\&A, 560, A50

Tsuneta, S., Ichimoto, K., Katsukawa, Y., et al. 2008, Sol. Phys., 249, 167

Wedemeyer, S., Freytag, B., Steffen, M., Ludwig, H.-G., \& Holweger, H. 2004, A\&A, 414, 1121 


\section{Appendix A: Additional figures}
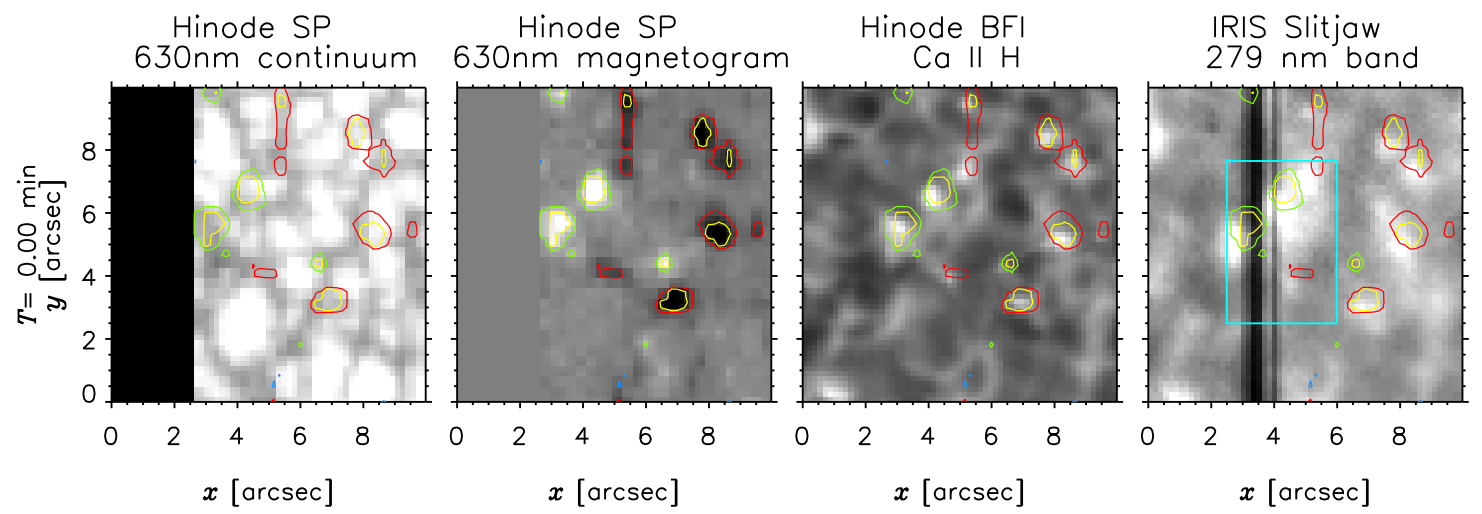

Fig. A.1. First panel: Hinode SP $6302 \AA$ continuum maps constructed from Stokes $I$ spectra. Part of the field-of-view is not covered by the SP slit data. The images were clipped between $10 \mathrm{k}$ and $16 \mathrm{k}$. The following panel displays the $B_{\text {long }}^{\text {app }}$ maps clipped at $\pm 80 \mathrm{Mx} \mathrm{cm}^{-2}$ retrieved with the sp_prep.pro code. This is followed by the Hinode Ca II H image (clipped to below 1500 counts) and the IRIS $2796 \AA$ band slitjaw image (clipped to below 200 counts). In the IRIS slitjaw images one can clearly see the IRIS slit position as a vertical dark strip. A thinner dark strip next to the slit position is due to reflections within the instrument. In all panels the contours are for $\pm B_{\text {long }}^{\text {app }} 30 \mathrm{Mx} \mathrm{cm}^{-2}$ shown with green and red colors denoting opposite polarity. The yellow contours are at levels of $60 \mathrm{Mx} \mathrm{cm}^{-2}$ shown for both polarities. The contours for $B_{\text {trans }}^{\text {app }} 120 \mathrm{Mx} \mathrm{cm}^{-2}$ are in blue. The FOV marked with a square in the last column shows the area displayed in Fig. 2. The times labeled on the left refer to the recording time of the IRIS slitjaw images in the $2796 \AA$ band. For Hinode we chose the closest frames referring to this time (see also Sect. 2 for explanation of the time frame). See online movie.
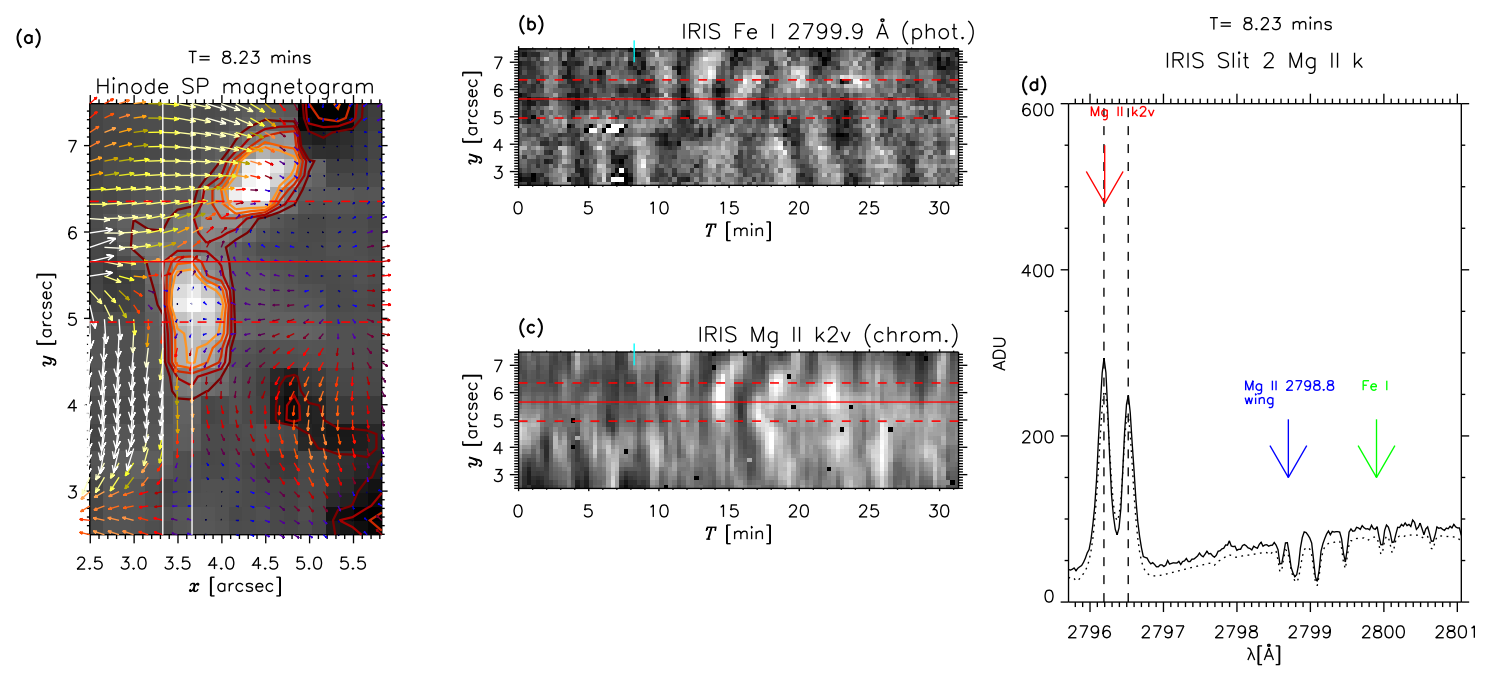

Fig. A.2. Panels a) to c): same as in Fig. 2. Panel a) shows the Hinode SP $B_{\text {long }}^{\text {app }}$ map now rebinned to the IRIS slitjaw scale with the same contours as in the second row of Fig. 1 and the horizontal flows indicated with colored arrows as in Fig. 1. The two vertical white lines delimit the position of the IRIS slit in use. Two dashed red horizontal lines and a solid red line mark a region of interest. Panels b) and c) are timespace images derived from spectra recorded by the second IRIS slit. Panel b) shows the line-core velocity of the Fe I $2799.9 \AA$ line $\left( \pm 1.9 \mathrm{~km} \mathrm{~s}{ }^{-1}\right.$, where down flows are positive) retrieved with the MOSiC code and in panel c) the intensity of the Mg II k2v peak (in data units) obtained by the iris_get_mg_features_lev2.pro code is plotted. The red horizontal lines indicate the same area as in panel a). The short vertical line in cyan indicates the time of the maps displayed in panel a). In panel d) the spectral profile recorded by IRIS is plotted with a solid line as well as an average quiet Sun spectrum (dotted). The dashed vertical lines denote the average position of the $\mathrm{Mg}$ II $\mathrm{k} 2 \mathrm{peaks}$. The arrows point to the Mg II $\mathrm{k} 2 \mathrm{v}$ peak, the wing of the Mg II $2798.8 \AA$ line, and the position of the Fe I $2799.9 \AA$ line used in panel b). See online movie. 